\title{
Lumen
}

Selected Proceedings from the Canadian Society for Eighteenth-Century Studies

Travaux choisis de la Société canadienne d'étude du dix-huitième siècle

\section{Images de Jean-Jacques : duplicité et liberté}

\section{Philip Knee}

Volume 20, 2001

URI : https://id.erudit.org/iderudit/1012308ar

DOI : https://doi.org/10.7202/1012308ar

Aller au sommaire du numéro

Éditeur(s)

Canadian Society for Eighteenth-Century Studies / Société canadienne d'étude du dix-huitième siècle

ISSN

1209-3696 (imprimé)

1927-8284 (numérique)

Découvrir la revue

Citer cet article

Knee, P. (2001). Images de Jean-Jacques : duplicité et liberté. Lumen, 20, 137-146. https://doi.org/10.7202/1012308ar

Copyright (c) Canadian Society for Eighteenth-Century Studies / Société canadienne d'étude du dix-huitième siècle, 2001
Ce document est protégé par la loi sur le droit d'auteur. L'utilisation des services d'Érudit (y compris la reproduction) est assujettie à sa politique d'utilisation que vous pouvez consulter en ligne.

https://apropos.erudit.org/fr/usagers/politique-dutilisation/ 


\section{Images de Jean-Jacques: duplicité et liberté}

Le problème de la duplicité dans la politique de Rousseau a suscité au fil des années de nombreux débats. Pour les ordonner un peu, je distinguerai, très schématiquement, deux approches ou deux images de Rousseau dans les études récentes, qui confèrent, par rapport à la grande thèse du Contrat social sur la légitimité politique, un statut différent aux textes où se manifeste cette duplicité. Par ceux-là, j'entends les chapitres du Contrat social sur le législateur et la religion civile (Livre II, 7 et Livre IV, 8), certains passages des écrits sur la Pologne et la Corse, les lettres sur la direction de Clarens par Wolmar dans la Nouvelle Héloïse ou encore la Lettre à D'Alembert sur les spectacles ${ }^{1}$.

Une interprétation qu'on peut appeler libérale voit dans le Contrat social une réponse au programme annoncé dans le Discours sur l'inégalité, où l'homme est défini comme un être perfectible, c'est-à-dire capable d'autodétermination. La politique de Rousseau est une politique de l'autonomie, centrée sur l'idée de loi, où la cité du contrat opère comme une Idée régulatrice (selon le vocabulaire kantien). Dans cette perspective, les textes sur la duplicité sont souvent négligés, par exemple chez Luc Ferry, qui s'appuie sur Rousseau comme inspirateur de la philosophie moderne de la liberté, mais en se référant avant tout à un passage du Discours surl'inégalité ${ }^{2}$; dans le même esprit, les chapitres du Contrat social mentionnés plus haut sont aussi interprétés comme des signes de l'échec de l'entreprise, des «béquilles», dit par exemple Alexis Philonenko. Dans une perspective proche, mais selon une méthode de lecture inverse, un commentateur comme Lester Crocker, préoccupé surtout par le totalitarisme il y a quelques décennies, s'est fait une spécialité de mettre en évidence et de dénoncer les passages les plus manipulateurs chez Rousseau, pour regretter en quelque sorte que cette duplicité contredise la teneur libérale de sa pensée ${ }^{4}$.

De l'autre côté, des commentateurs d'inspiration straussienne, comme Allan Bloom, mettent eux aussi en évidence tout ce qui relève chez Rousseau de stratégies secrètes, mais pour s'en féliciter au contraire, en inscrivant Rousseau dans la tradition de l'écriture ésotérique ou des nobles mensonges de Platon ${ }^{5}$. Dans cette ligne, la clé de la politique de 
Rousseau réside, non dans la cité du Contrat social, mais dans la formation de l'imagination des citoyens et dans la fabrication de leur sentiment d'appartenance, en regard de quoi le contrat lui-même n'est qu'une «fiction tautologique», selon l'expression de Pierre Manent ${ }^{6}$, une fiction qui formule juridiquement le résultat d'un processus essentiellement psychologique. Une telle politique, axée sur une duplicité nécessaire au bonheur des hommes et à l'ordre de la cité, semble alors étrangère à l'idéal de liberté, s'il est compris dans le sens moderne de l'autonomie.

Mais on peut aussi s'intéresser à cette question dans une perspective assez différente de ces deux approches: moins pour dénoncer les manœuvres politiques préconisées par Rousseau ou pour en faire l'éloge, que pour mieux comprendre, grâce à elles, le fonctionnement de nos sociétés démocratiques. À l'instar des moralistes du XVII siècle qui décrivent les jeux de masques comme une dimension irréductible des rapports interindividuels, on peut tenir pour acquis qu'une certaine duplicité est inscrite dans toute vie politique et s'efforcer de distinguer entre les formes de cette duplicité selon leurs effets ou leurs intentions. $C^{\prime}$ est ce que fait par exemple un livre récent de Ruth Grant ${ }^{7}$, même s'il se situe globalement dans une perspective libérale. Il récuse, non seulement toute condamnation moraliste de la duplicité, mais aussi l'idée d'une vie politique conçue sur le modèle des rapports commerciaux, où pourrait régner ouvertement le calcul d'intérêt et dont pourraient être ainsi exclus toute hypocrisie et donc tout besoin de duplicité, grâce à une négociation rationnelle des différences au bénéfice de tous. Cherchant à définir une prise en charge limitée de la duplicité, cette démarche de Grant me semble la plus féconde pour aborder les paradoxes de la politique de Rousseau; elle inspirera ma tentative ici, non de décrire les figures de la duplicité qui se déploient dans son œuvre (je me limiterai à une brève évocation du cas du Législateur $)^{8}$, mais de cerner avec plus de précision qu'on ne le fait habituellement l'articulation de la duplicité et de la liberté dans sa pensée.

S'il est bien lu, on voit que le Discours sur l'inégalité met en place l'enjeu de la duplicité en traitant la question de l'inégalité. Rousseau s'y propose de combattre l'inégalité morale, qu'il comprend dans le sens précis de la dépendance où se trouve placée la masse des hommes bernés par le faux contrat des puissants ${ }^{9}$. À côté de cela, il y a donc des inégalités légitimes: celles qui concourent "en même proportion avec l'inégalité physique», dit-il, comme les "différences d'âge, de santé, de force du corps, des qualités de l'esprit et de l'âme ${ }^{10}{ }$. Rousseau en donne pour exemples la supériorité du vieillard sur l'enfant ou du sage sur l'imbécile. Le cœur consent à ces inégalités comme il consent aux inégalités physiques. Ce ne sont donc ni toutes les inégalités ni même toutes les dépendances qui 
sont combattues. Si l'homme doit s'arracher à certaines dépendances pour assurer sa liberté, il doit savoir se soumettre à d'autres.

Comment distinguer la bonne de la mauvaise dépendance? Il y a certes des critères qu'on peut appeler objectifs, puisque certaines dépendances, comme l'esclavage, sont des négations de l'humanité de $l^{\prime}$ homme. Mais il ne suffit pas qu'une dépendance soit objectivement bonne pour qu'elle concoure à la liberté. Elle doit être vécue d'une certaine manière: en évitant certains effets moraux du sentiment de dépendance qui sont suscités par l'amour-propre dans la société corrompue. La dépendance peut être libérée d'une expérience subjective destructrice si elle n'est pas vécue comme une dépendance sur d'autres volontés personnelles et identifiables, car dans ce cas l'hypocrisie, l'envie, le ressentiment n'ont pas de prise sur l'homme.

Ce principe est à l'œuvre dans le Contrat social. La liberté y consiste à vivre sous l'autorité des lois, en s'assurant que le peuple puisse ratifier ces lois et s'y reconnaître. Mais pour cela il doit être formé à ne plus les voir comme une dépendance sur le pouvoir d'autres hommes, et cela passe par une ruse: que le peuple les voie comme ayant une origine divine. Y consentir sur cette base illusoire rend les hommes libres. Rousseau pourrait dire qu'y consentir ainsi est rationnel, car c'est un moyen efficace de réaliser la fin recherchée: s'obéir à soi-même. Mais ce n'est pas un acte rationnel dans le sens d'un acte délibéré, conscient, guidé par la raison - au contraire, puisque les hommes consentent à ce qu'ils croient être des commandements divins, alors que les lois sont d'origine humaine. Cette dépendance dissimulée ou impersonnelle ne produit pas d'effets nocifs. L'homme s'y sent proche de son état originel ou de son essence, il y retrouve le goût de son indépendance naturelle. Mais en vérité cette expérience n'est pas celle de l'indépendance, puisqu'elle dépend de ceux qui la façonnent.

Formulons le problème tel qu'il se présente dans l'action du Législateur. Située entre les mœurs et la loi, là où s'articulent la raison et la passion des hommes, cette action doit combiner l'exigence de légitimité, telle que manifestée par les principes abstraits du droit politique, et l'exigence d'inscrire le consentement à ce qui découle de ces principes dans le cœur des citoyens. Faire des lois consiste d'abord à constituer une volonté générale capable de vivre conformément à elles, et pour cela il faut dominer les opinions et par elles gouverner les passions des hommes, en agissant sur les usages, les cérémonies religieuses, les spectacles qui rappellent l'histoire des peuples, etc. Si cette action réussit, le peuple se reconnaît dans les dispositions prises par le Législateur, et celles-ci lui semblent naturelles même s'il ne partage pas la science qui y a présidé. 
Or, pour que le peuple sente comment les choses doivent être, et ne sente que par le tout, le Législateur doit "persuader sans convaincre ${ }^{11}$ », c'est-à-dire utiliser le langage du cœur, non celui de la raison. Certes, la volonté générale n'existe qu'à condition que chacun concoure à la dégager et se prononce sur un projet législatif; mais cette volonté ne saurait se constituer que si le peuple (qui cherche le bien, mais ne l'aperçoit pas toujours) est amené à voir les objets «tels qu'ils doivent lui paraître ${ }^{12}{ }^{\prime}$. Ignorer la persuasion, c'est-à-dire le langage qui affecte l'âme, c'est ignorer ce qui fait l'essentiel de la vie politique et c'est se condamner, à terme, à faire usage de la force pour assurer l'ordre. Voilà à quoi sont conduits les hommes politiques modernes, selon Rousseau, avec leur langage adressé à la raison et destiné seulement à convaincre. Utilisant des «langues favorables à la liberté» et faites pour la place publique, la politique des Anciens reposait au contraire sur la persuasion. Avec leurs langues «faites pour le bourdonnement des divans», la politique des Modernes est obligée de faire que «la force publique supplée à la persuasion ${ }^{13}{ }^{\prime}$.

Cependant, pour agir sur les sentiments, le Législateur ne fait pas que parler: il inspire. Rousseau souligne le rôle que jouent aussi chez les Anciens les gestes qui frappent l'imagination, par exemple, l'effroi salutaire provoqué par Brutus mettant à mort ses propres enfants après que ceux-ci eurent conspiré contre l'État ${ }^{14}$. Dans ce cas, le Législateur persuade en offrant au peuple l'exemple de sa grandeur d'âme, car il cherche moins à démontrer pour que le peuple voie qu'à se montrer de telle façon que le peuple sente. Il s'agit de réunir dans son action les deux composantes qui faisaient le succès de la petite communauté de Clarens dans la Nouvelle Héloïse, où l'exemple de vertu de Julie et le paternalisme de Wolmar se complétaient. Cette méthode se manifeste aussi dans l'utilisation politique que le Législateur fait de la religion: il met ses paroles dans la bouche des dieux, certes; mais surtout il se présente de manière à être perçu comme un médiateur entre le peuple et les dieux, une figure digne de susciter de cette manière le goût d'être imitée.

Ne pouvant convaincre un peuple non encore institué, le Législateur doit donc avoir recours à la ruse, à un entraînement par les sentiments, à un maniement de l'illusion et de l'image afin de faire jouer en secret l'opinion. Parfait connaisseur des ressorts cachés de la nature humaine, il doit savoir se placer au-dessus de l'opinion pour gouverner les hommes par elle; faire que les citoyens soient amenés à réaliser les fins rationnelles par eux-mêmes, en étant mis dans des situations où ils reçoivent des objectifs pour leur volonté sans le savoir. Si Rousseau n'hésite pas à appeler libre cette volonté manipulée mais droite, on voit que le terme recouvre une ambiguïté. D'un côté, il s'agit des croyances des hommes utilisées par le Législateur pour agir sur eux dans la mesure 
où ils sont soumis à l'opinion, c'est-à-dire à l'image de liberté que leur renvoient les autres hommes. De l'autre, il s'agit de la valeur d'une conduite soumise à la loi du Législateur en tant que celle-ci incarne, à travers la ruse qui l'impose, la raison. Le citoyen n'est soumis à la volonté de personne, mais sa propre volonté n'est constituée que par celle du Législateur. Il intériorise cette autorité par laquelle sa volonté prend forme, et c'est cette intériorisation qui le fait libre. Sa liberté ne consiste pas à faire des lois à travers une délibération avec ses concitoyens, mais à vivre selon les lois qui conviennent, en y conformant sa volonté; et c'est cette volonté que doit produire le Législateur. Celui-ci doit donc créer des patriotes et des hommes vertueux, mais pas des législateurs. En d'autres termes, le citoyen libre doit être actif sur le plan du sentiment d'appartenance, mais passif sur celui de la fabrication ou du changement des lois ${ }^{15}$. Par cette liberté strictement limitée, on voit que Rousseau établit, non un simple décalage entre l'entendement du peuple et celui du Législateur, mais une différence de nature que rien ne peut venir combler: le peuple reste un enfant incapable d'user rationnellement de sa liberté.

La plus grande partie du Contrat social décrit la liberté politique en la situant dans le cadre de l'illusion subjective qui lui est nécessaire. Les autres textes politiques et les développements du Contrat social sur le Législateur et la religion civile décrivent les moyens de la fabrication de cette illusion. La question que pose cette vision dédoublée de la politique est la suivante: le citoyen peut-il vivre ensemble ces deux aspects de la politique? Peut-il lier le discours juridique qui procède de l'illusion politique sans la montrer comme illusion, avec le discours sur la fabrication de cette illusion et sa nécessité? En d'autres termes, est-il possible de faire simultanément l'expérience de l'ordre politique comme un ordre moralement légitime, car garantissant la liberté des citoyens, et l'expérience de l'ordre politique comme un ordre fabriqué par des législateurs pour les citoyens à l'insu de ces derniers?

Sans la formuler de manière explicite, les textes de Rousseau ne cessent de mettre en évidence cette question. On aperçoit bien son enjeu psychologique par un exemple tiré de la partie non directement politique de l'œuvre, à l'une des étapes de l'éducation d'Émile. L'entente passée, à la naissance de l'enfant, entre le gouverneur et son père, et légitimant l'autorité absolue du gouverneur, est révélée à Émile au Livre IV, c'està-dire au début de son adolescence. Jusque-là, il fallait le préserver de cette révélation, car, trop jeune, il y aurait mal réagi: il n'aurait pas compris que l'abdication de sa volonté et de sa liberté que lui imposaient les ruses du gouverneur était à son avantage. Il n'était capable de juger des choses que selon les lois de la nature, pour lesquelles l'assujettissement à autre chose que la nécessité naturelle est inacceptable. Pour 
l'accepter, il lui aurait fallu comprendre que, dans un monde corrompu, il faut être préparé à vivre en étant préservé des effets de cette corruption. Cette période où on le préserve d'une vérité qu'il ne peut entendre est celle de l'éducation dite négative. En fait, contrairement à ce qu'on dit souvent, c'est celle d'un contrôle total du gouverneur sur l'enfant, celle du règne de la duplicité.

Au Livre IV, on passe à l'éducation positive, adaptée à la naissance de la raison et de la conscience morale d'Émile, quand le rôle du gouverneur peut être dévoilé. Comment celui-ci s'y prend-il? Avant tout en montrant à Émile qu'il a agi jusque-là par affection ${ }^{16}$. Il lui révèle le contrat de départ et lui propose un nouveau contrat par lequel Émile consent lui-même, cette fois, à se soumettre à l'autorité du gouverneur. L'engagement du gouverneur à continuer à agir pour le bien d'Émile est rendu crédible par son action passée. On voit le paradoxe: le gouverneur demande à Émile de croire en sa sincérité, alors qu'il lui révèle qu'il a été insincère depuis quinze ans. Émile ne peut y consentir que dans la mesure où il relit son passé à la lumière de ce qu'il vient d'apprendre et qu'il constate que le monde illusoire dans lequel on l'a fait vivre correspond à son bien et procède de l'affection du gouverneur, non de ses intérêts ou de son goût du pouvoir.

Dans ce moment privilégié se concentrent les deux exigences indiquées plus haut: exister dans un monde fictif qui est la condition de la liberté; consentir à cette fiction et au fait qu'elle est entretenue par la duplicité. Le lecteur des deux faces de la politique de Rousseau est invité, comme Émile, à reconnaître que la duplicité est indispensable et qu'elle se justifie par une visée qui la dépasse. La liberté exige qu'on gouverne les hommes par le consentement, mais ce consentement peut être un consentement par la duplicité (comme chez Émile enfant) ou un consentement à la duplicité (comme chez Émile adolescent). Les deux approches sont nécessaires, car elles correspondent à la présence dans la société de types d'hommes différents, ou en tout cas d'états psychologiques différents par lesquels passent les hommes. C'est pourquoi Rousseau ne tranche jamais clairement entre deux conceptions politiques classiques ${ }^{17}$ - celle d'une autorité fondée sur la sagesse des gouvernants et celle d'une autorité fondée sur le consentement des gouvernés - car la sagesse est présentée comme la capacité de gagner le consentement par la duplicité.

«L'obéissance à la loi qu'on s'est prescrite est liberté», écrit Rousseau dans le Contrat social ${ }^{18}$. On a vu que cette obéissance était forgée par la puissance éducative de lois données par un Législateur; que la liberté politique était bâtie par des moyens qui semblent la contredire. Rousseau écrit par ailleurs: «Par où donc émouvoir les cœurs et faire aimer la patrie et les lois? L'oserai-je dire? Par des jeux d'enfants; par des institutions 
oiseuses aux yeux des hommes superficiels, mais qui forment des habitudes chéries et des attachements invincibles ${ }^{19}$.» Comment comprendre l'articulation de ces deux citations? En fin de compte, la liberté réside-telle dans ce patriotisme, dans ces habitudes et ces attachements, ou dans l'obéissance à la loi qu'on s'est prescrite, c'est-à-dire dans l'autonomie? Rousseau ne répond pas nettement, car il ne se demande jamais explicitement si un consentement politique acquis par la duplicité met en cause la liberté. Il procède plutôt comme si la liberté n'était accessible que par la duplicité. Les deux perspectives sont ainsi entremêlées.

Les penseurs libéraux comme Locke, qui font du consentement la condition de la légitimité politique, admettent que le consentement ne peut toujours être un acte délibéré; il y a des formes plus faibles, des consentements tacites, qui ne rendent pas pour autant illégitimes les gouvernements dont ils sont $\mathrm{l}^{\prime}$ objet $^{20}$. Mais ces penseurs font néanmoins du choix raisonné des citoyens, le plus explicite possible, la visée de la politique. Ce critère d'un consentement délibéré est décisif pour juger du sens de la politique de Rousseau quant à la liberté. Non le critère de la présence ou de l'absence de duplicité; mais le fait que plus de rationalité ne signifie pas, pour lui, plus de liberté; que plus d'honnêteté politique ou moins de duplicité ne représente pas, pour lui, un progrès moral. Une traduction politique de l'éducation d'Émile impliquerait de viser une société qui parviendrait, comme l'enfant, à une sorte de maturité, rendant la duplicité inutile; mais Rousseau n'envisage nullement une telle généralisation à tous de l'éducation individuelle.

Il faut donc distinguer la duplicité du Législateur de celle du gouverneur d'Émile, car si ce dernier utilise tout autant une technique du paraître et s'il truque ses rapports avec son élève, on a vu que c'est pour permettre à cet élève de conquérir son indépendance, de se défaire de toute soumission. L'établissement de l'objectif à atteindre dépend du diagnostic posé sur la condition morale et psychologique des hommes auxquels on a affaire, tout comme l'état de la société genevoise ou parisienne décide, dans la Lettre à D'Alembert par exemple, de la teneur de l'attaque de Rousseau contre les spectacles. La duplicité est nécessaire dans tous les cas, mais selon des modalités et des finalités différentes. Dans un cas, c'est pour utiliser les effets de cette duplicité sans espoir $d^{\prime}$ 'enlever les masques; dans l'autre, c'est pour faire prendre conscience, à terme, de la fonction salutaire de cette duplicité. Mais ces différences de diagnostic quant au degré de dénaturation et donc au dosage de duplicité nécessaire semblent recouvrir une distinction plus générale entre l'individu et le peuple dans leur rapport à l'opinion. Il manque aux peuples la possibilité même de la maîtrise de leurs passions telle que peuvent la développer certains individus à partir de leurs virtualités. Les peuples sont et restent des enfants, même si ces enfances peuvent être 
plus ou moins réformables (comme à Genève, en Pologne ou en Corse). Ainsi la politique de Rousseau fige les principes de duplicité de sa pédagogie, non dans la perspective d'un devenir-adulte, mais dans celle de la gestion rationnelle du goût des images inhérent aux peuples.

On a parfois avancé que, dans l'économie générale de la pensée de Rousseau, l'éducation de l'homme et celle du citoyen devaient être comprises comme complémentaires, les institutions politiques du Contrat social et la maturité individuelle d'Émile étant requises l'une par l'autre pour constituer une éthique sociale cohérente ${ }^{21}$. Mais l'articulation des deux composantes ne se trouve pas explicitement chez Rousseau, car sa politique ne ménage pas la possibilité d'une mobilité intellectuelle et morale telle que l'Émile en dessine les conditions. On peut en conclure qu'une telle perspective n'a de sens que réservée à des individus d'exception chargés de manier la ruse pour agir sur l'opinion, en ayant pris conscience du défaut rédhibitoire qui afflige l'existence collective. C'est à certains individus que reviendrait la tâche de pallier la carence du peuple, certains hommes étant les objets de la politique, d'autres pensant la politique et y échappant. Dans cette perspective, qu'on peut appeler platonicienne, Rousseau serait un critique féroce des idéaux démocratiques modernes. Seuls les lecteurs qui savent embrasser la totalité du projet de Rousseau, avec ses divers niveaux de duplicité, seraient destinés, à leur tour, à faire œuvre éducatrice ou législatrice. On peut ainsi imaginer Émile, après son retour de voyage et l'achèvement de son éducation, s'engageant dans la vie de sa cité (c'est-à-dire retournant dans la caverne, selon l'image de la République) et maniant à son tour la duplicité pour le bien des autres hommes.

Mais Émile n'a pas été formé pour être un législateur et Rousseau laisse entendre qu'il préfère rester à l'écart de la chose publique ${ }^{22}$; de plus, ce qu'on apprend de sa vie d'adulte montre sans doute les effets positifs de son éducation, mais n'en révèle pas moins des faiblesses et des échecs ${ }^{23}$. Aussi, comme je l'ai suggéré plus haut, peut-être faut-il être surtout attentif à la période qui précède cet achèvement de l'éducation d'Émile, celle de son adolescence et de son mariage, où il consent à rester sous l'autorité du gouverneur, afin que son éducation par la duplicité se poursuive, alors même qu'elle lui a été révélée. Car cette expérience permet d'envisager une vie politique qui serait au fait de la duplicité qui l'anime, et cette figure me semble pertinente pour réfléchir sur notre vie démocratique aujourd'hui. Plutôt que d'opposer le Rousseau libéral au Rousseau platonicien, on peut chercher à mettre cette ambivalence au service d'une meilleure intelligence de certains aspects problématiques de notre conscience moderne, en déplaçant ainsi l'attention de la duplicité des gouvernants à celle des gouvernés. En effet, tout se passe comme si les citoyens que nous sommes aspiraient à vivre en même 
temps les deux faces de la politique de Rousseau, en dévoilant la duplicité et en reconnaissant sa nécessité; en tenant à la fois un discours de liberté et de justice manifestant un ordre de croyances qui enveloppe les citoyens et un discours qui démystifie ces croyances et révèle leur fabrication. Quelques décennies après ces textes de Rousseau, lorsque Tocqueville, par exemple, observe en Amérique ce qu'il appelle le patriotisme réfléchi et la religion utilitaire de l'homme démocratique, et qu'il s'interroge sur la nature de cette appartenance et de cette foi ${ }^{24}$, n'est-ce $^{\prime}$ pas précisément le fonctionnement d'une telle conscience dédoublée qu'il s'efforce de penser?

En donnant une place irréductible à la duplicité, Rousseau rejette une politique libérale ou utilitariste, si l'on entend par là qu'elle vise un ordre politique où régnerait un débat public régi par la seule raison. Mais il n'est pas pour autant machiavélien, si l'on entend par là qu'aucune valeur n'échappe à la politique et que la liberté n'est qu'un vain mot hors des enjeux du pouvoir. La duplicité est inéliminable dans la mesure où l'ordre politique est un ordre de dépendances et d'apparences, auquel les hommes ne sauraient échapper radicalement; mais c'est un ordre dont ils ne sont pas pour autant les dupes à tout moment. Cette image instable, inconfortable de la politique de Rousseau me semble pouvoir être utilement explorée davantage.

\section{PHILIP KNEE}

\section{Notes}

1 Toutes les références aux textes de Rousseau renvoient à l'édition des CEuvres complètes, en cinq tomes, sous la direction de Bernard Gagnebin et Marcel Raymond, Paris, Gallimard, coll. «Bibliothèque de la Pléiade», 1959-1995.

2 Voir le début de l'ouvrage de Luc Ferry, l'Homme Dieu ou le sens de la vie, Paris, Grasset, 1996, p. 13-14.

3 Alexis Philonenko, Jean-Jacques Rousseau et la pensée du malheur, tome III: Apothéose $d u$ désespoir, Paris, Vrin, 1984, p. 81.

4 Voir notamment les articles de Lester Crocker, «Rousseau et l'opinion», Studies on Voltaire and the Eighteenth Century, 55, 1962 et «Julie ou la nouvelle duplicité», Annales de la Société Jean-Jacques Rousseau, 36, 1963-1965.

5 De Léo Strauss lui-même, voir «L'intention de Rousseau», dans Gérard Genette et Tzvetan Todorov (édit.), Pensée de Rousseau, Paris, Seuil, 1984. Voir aussi Roger Masters, The Political Philosophy of Rousseau, Princeton, Princeton University Press, 1968 et Allan Bloom, "Introduction», dans Émile, or On Education, New York, Basic Books, 1979.

6 Pierre Manent, Naissances de la politique moderne, Paris, Payot, 1977, p. 172. 
7 Ruth Grant, Integrity and Hypocrisy. Machiavelli, Rousseau and the Ethics of Politics, Chicago et Londres, University of Chicago Press, 1997.

8 Pour une description plus complète, notamment du paternalisme de Wolmar, je me permets de renvoyer à mon ouvrage Penser l'appartenance. Enjeux des Lumières en France, Montréal, Presses de l'Université du Québec, 1995, chapitre II.

9 Discours sur l'inégalité, O.C., tome III, p. 177-178.

10 Discours sur l'inégalité, O.C., tome III, p. 47 et 194.

11 Du contrat social, O.C., tome III, p. 383.

12 Du contrat social, O.C., tome III, p. 380.

13 Essai sur l'origine des langues, O.C., tome V, p. 428-429.

14 Dernière réponse à Bordes, O.C., tome III, p. 88.

15 Décrivant la cité dans laquelle il aurait aimé vivre, Rousseau est très clair à ce sujet dès la dédicace du Discours sur l'inégalité, O.C., tome III, p. 114.

16 Émile, O.C., tome IV, p. 648.

17 Elles correspondent grosso modo aux deux interprétations de sa pensée que j'ai évoquées au début de ce texte.

18 Du contrat social, O.C., tome III, p. 365.

19 Considérations sur le gouvernement de Pologne, O.C., tome III, p. 955.

20 John Locke, Traité du gouvernement civil, Paris, Garnier-Flammarion, 1984, $\S 119-122$.

21 Voir par exemple l'ouvrage de Tzvetan Todorov, Frêle bonheur. Essai sur Rousseau, Paris, Hachette, 1985.

22 Voir Émile, O.C., tome IV, p. 855-860.

23 Voir ce récit dans les deux lettres d'Émile et Sophie ou les solitaires, O.C., tome IV.

24 Alexis de Tocqueville, De la démocratie en Amérique I, Paris, Robert Laffont, 1986, $2^{\mathrm{e}}$ partie, notamment les chapitres 6 et 9 . 